\title{
El Telebachillerato Comunitario. Una innovación curricular a discusión
}

\author{
Telebachillerato comunitario: \\ A Curriculum Innovation under Discussion
}

\begin{abstract}
Eduardo Weiss, * con la colaboración de Lilia Antonio, Enrique Bernal,
Cyntia Guzmán y Paola Pedroza
\end{abstract}

\section{RESUMEN}

En el contexto de la obligatoriedad de la educación media superior se estableció a partir de agosto de 2013 un nuevo tipo de servicio educativo, el Telebachillerato Comunitario, que busca atender a los jóvenes que habitan en poblaciones rurales de hasta 2500 habitantes. En un estudio exploratorio de su puesta en práctica en cinco estados de la República analizamos sobre todo las estructuras institucionales del currículum. Este servicio trabaja, mayoritariamente en las tardes, en instalaciones de las telesecundarias con tres docentes. El aprendizaje se basa en libros de texto gratuitos. El servicio es aceptado por las familias por su cercanía y la mayor seguridad en los caminos y por su gratuidad efectiva. Una innovación curricular interesante es que los tres docentes no son contratados por asignatura sino por campo disciplinar: matemáticas y ciencias experimentales; comunicación; ciencias sociales y humanidades. Las tensiones principales que detectamos entre el modelo y su operación son: a) la ubicación de las escuelas en instalaciones de telesecundarias y en turno vespertino, b) la sobrecarga de trabajo para tres docentes; $c$ ) las tensiones en el diseño curricular, en especial entre un modelo que promueve el aprendizaje a partir de proyectos comunitarios y el plan de estudios tradicional de bachillerato general de tipo colección de asignaturas académicas en la tradición positivista que privilegia los conocimientos abstractos.

Palabras clave: educación media superior, educación rural, educación comunitaria, currículum, México

\section{ABSTRACT}

In the context of compulsory high school education, a new type of educational service was established in 2013: the "Telebachillerato Comunitario" (Community High School with Video-based Learning), in an attempt to reach young people living in rural populations of up to 2500 inhabitants. Based on an exploratory study of its implementation in five states of Mexico, we focused our analysis on the institutional structures of the curriculum. We found that this service functions in most cases in the afternoon, in tele-secondary facilities with three teachers and learning is based on free of cost textbooks. It is accepted by families due to the proximity, the increased safety of roads, and the schools' cost-free nature. An interesting innovation in the curriculum is that the three teachers are not hired by subject, but by disciplinary field: mathematics and experimental science; communication; and social science and humanities. The primary tensions that we detected between the model and its operation are: a) the schools' location in telesecondary facilities in the afternoon; $b$ ) the excess workload for the three teachers; and c) tensions in curriculum design, especially between a model that promotes learning based on community projects, and the usual study plan of general high school, consisting of a collection of academic subjects ordered in the positivist tradition, with an emphasis on abstract knowledge.

Key words: high school education, rural education, community education, curriculum, Mexico

\footnotetext{
Departamento de Investigaciones Educativas del Centro de Investigación y de Estudios Avanzados del IPN (DIE-CINVESTAV), eweiss@cinvestav.mx
} 


\section{El Telebachillerato Comunitario: su marco político y normativo}

Hace más de una década, el Estado mexicano decidió mejorar sustancialmente la educación media superior. En 2005 se creó la Subsecretaría de Educación Media Superior (SEMS), que reunía las direcciones generales que antes formaban parte de la Subsecretaría de Educación Superior y de la Subsecretaría de Educación e Investigación Tecnológicas; en 2008 se estableció un marco curricular común basado en competencias y la Reforma Constitucional del 9 de febrero de 2012 instituyó la obligatoriedad de la educación media superior. El carácter obligatorio establece como deber del Estado ofrecer un lugar a todos los jóvenes que, teniendo la edad típica para cursarla, hayan concluido la educación básica. Se estipuló que esta obligatoriedad se realizará de manera gradual y creciente a partir del ciclo escolar 2012-2013, hasta lograr la cobertura total en sus diversas modalidades en el país, a más tardar en el ciclo escolar 2021-2022 (Diario Oficial de la Federación, 9 de febrero de 2012).

La educación media superior mexicana se había desarrollado por encima del crecimiento poblacional de 2.1 millones de alumnos en 1990-91, a 4.7 millones en 2012-13, de manera que la cobertura aumentó de 34.1\% en 1990-91 a 65.9\% en 2011-2012. No obstante, un tercio de los jóvenes en ese rango de edad aún no podía cursar la educación media superior, además de que representa una tasa de escolarización muy por debajo de la de países comparables como Chile y Argentina, donde los jóvenes de 15, 16 y 17 ańos en 2010 habían ya alcanzado tasas de escolarización de entre $82.5 \%$ y $96.3 \%$ (SITEAL, 2014: tabla 5).

En el Plan Nacional de Desarrollo 2012-2018 se estableció la meta de alcanzar 80\% de cobertura en este nivel (SEMS, 2015: 6). La Subsecretaría de Educación Media Superior instrumentó varias medidas para lograrlo: un financiamiento concursable para la inversión en infraestructura que permite construir nuevos planteles y ampliar los ya existentes, el crecimiento de la planta docente en planteles nuevos o ampliados con recursos del Fondo de Ampliación de la Cobertura, el crecimiento de la planta docente en planteles a través del contraturno, la creación o fortalecimiento 
de servicios como el Telebachillerato Comunitario, la preparatoria abierta y la prepa en línea (CONAEDU, 2015), así como con la política de becas y la de "cerrar la llave de abandono" a través del Movimiento contra el Abandono Escolar (Weiss, 2015; Pérez Islas et al., 2016). En el ciclo escolar 2015-2016, la educación media superior alcanzó una cobertura en la modalidad escolarizada de $74.2 \%$ de la población de 15 a 17 años. Si se toma en cuenta la matrícula de la educación media superior no escolarizada (i.e. el programa Prepa en Línea-SEP y la Preparatoria Abierta), la matrícula total es cercana a 5.3 millones de estudiantes, con lo que se alcanza una cobertura de 78.7\%. (SEP, 2016: 17).

En México han sido excluidos del acceso a la educación media superior grandes sectores de la población rural, de las zonas marginadas y de los grupos con ingresos y niveles de escolaridad más bajos. Los jóvenes de 15 a 17 años que hoy cursan la educación media superior y que habitan en zonas rurales representan $36 \%$, en contraste con $60 \%$ de los de las zonas urbanas. Quienes hablan una lengua indígena representan $29 \%$, en contraste con $54.5 \%$ de hablantes de espańol (INEE, 2011). Un estudio de la Subsecretaría de Educación Media Superior encontró que de las 16174 escuelas telesecundarias en localidades rurales, 7682 no contaban con un servicio de Educación Media Superior en $5 \mathrm{~km}$ a la redonda (SEMS, 2015: 6).

En este contexto, se estableció el Telebachillerato Comunitario con las siguientes características (SEMS, 2015: 8).

- Se busca atender a los jóvenes que habitan en poblaciones rurales de hasta 2500 habitantes y que no son atendidos por ninguna modalidad de los subsistemas (en documentos anteriores a 2015 se habla también de comunidades con menos de 1500 habitantes).

- Se establece preferentemente en las instalaciones de las Telesecundarias o en espacios que la propia comunidad ponga a disposición del servicio.

- Se imparte en una modalidad escolarizada presencial.

- Se sustenta en el plan de estudios del Bachillerato General.

- Cuenta con una plantilla de tres docentes.

- Se apoya en materiales impresos y audiovisuales elaborados expresamente para este servicio. 
- La duración de los estudios es de un mínimo de tres y un máximo de cinco años.

Esta propuesta inició en agosto de 2013 en una etapa piloto con 253 centros; en 2014 se incrementó en 1497 más y en agosto de 2015 se abrieron 1168 , teniendo un total de 2918 centros que atienden a una matrícula de, aproximadamente, 104 mil estudiantes, donde participan 8859 docentes (SEMS, 2015: 6).

\section{Un estudio exploratorio: método}

En septiembre de 2015, el Instituto Nacional para la Evaluación de la Educación (INEE) encargó al Departamento de Investigaciones Educativas del Centro de Investigación y de Estudios Avanzados (DIE-CINVESTAV) un estudio exploratorio denominado oficialmente "Estudio evaluativo del modelo de telebachillerato comunitario".

El estudio se realizó a partir del análisis de documentos y materiales producidos por el Telebachillerato Comunitario (TBC) y cinco estudios estatales.

Se seleccionaron las siguientes entidades: el Estado de México, por ser la entidad que cuenta con más TBCs en el país; Veracruz, por ser el estado cuna de los Telebachilleratos estatales, que ahora cuenta también con TBCs; Chihuahua, por contar una oferta diversificada para alumnos de localidades rurales, al tener números apreciables de TBCs, Telebachilleratos Estatales y Bachilleratos Interculturales; Guanajuato, por tener números sustanciales de TBCs y un TB estatal (SABES) importante y, finalmente, Aguascalientes, por contar (al igual que Yucatán) con los índices más altos de alumnos por TBC, siendo un estado bien comunicado y eminentemente urbano.

En los cinco estados se realizaron entrevistas a funcionarios responsables de este servicio educativo en la entidad, así como visitas a planteles: tres en Aguascalientes, tres en Chihuahua, seis en el Estado de México, dos en Guanajuato y dos en Veracruz. En las visitas se observaron algunas clases y fueron entrevistados los docentes (uno de ellos encargado de la organización del plantel), los alumnos de los diferentes grados y algunos familiares presentes. 
Las visitas de tres días a los estados de México, Veracruz y Chihuahua fueron realizadas por el responsable de este estudio (Dr. Eduardo Weiss) y personal comisionado y contratado para el proyecto (M. en C. Lilia Antonio y M. en C. Enrique Bernal); para realizar el estudio de Aguascalientes fue contratada la Mtra. Cintya Guzmán Ramírez y para el estudio de Guanajuato, la Antropóloga Paola Pedroza Obregón.

El informe final se entregó en febrero de 2016 (Weiss, 2016). Aquí se presentan los hallazgos principales referidos al Telebachillerato Comunitario dentro de un marco de referentes teóricos.

\section{Referentes teóricos: El plan de estudios y las estructuras institucionales del currículo}

Al establecer la obligatoriedad de la educación media superior, se está produciendo en todos los países de América Latina un cambio -no sin tensiones- de definición respecto a su función. La media superior dejó de ser preparatoria para la educación superior o formativa para el trabajo, para comenzar a concebirla como "parte constitutiva de la educación fundamental que todo ciudadano debiera poseer, y ya no como una situación excepcional o de privilegio" (Bellei, 2012: 220). No solo para los nuevos sectores sociales a incorporarse a la educación media superior, sino también para las nuevas generaciones, se pone en entredicho la relevancia de los estudios (Tenti, 2012). Hay que transformar las estructuras institucionales que obstaculizan el desarrollo de experiencias escolares sólidas, extensas y densas en aprendizajes significativos (Banks, 2007).

La posibilidad de mejorar la relevancia de los contenidos del currículo se relaciona directamente con una disminución del contenido informativo de los programas (lo que fue definido en este trabajo como enciclopedismo) y con la mejora de las conexiones de las diferentes materias con la contemporaneidad (Terigi, 2012: 70).

En México, la Reforma Integral de la Educación Media Superior propuso un nuevo discurso basado en el desarrollo de competencias, pero los planes de estudio cambiaron muy poco y en 
la mayoría de los programas solo se añadieron recomendaciones para fortalecer las competencias y siguieron con su enciclopedismo (Zorrilla, 2012).

La "gramática escolar" como la llaman Tyack y Tobin (1998) -o "cultura escolar" como la llama Viñao (2002) - de la educación secundaria (incluyendo la media superior) se basa en las asignaturas heredadas de fines del siglo XIX y principios del XX y en profesores especialistas contratados para impartir estas asignaturas, así como en la fragmentación del tiempo de enseñanza y de aprendizaje en un cúmulo de periodos cortos (Quiroz, 1992). Bernstein (cf. Sadovnik, 2001) los ha llamado currículos clasificados de colección y ha propuesto sustituirlos por currículos integrados y, desde tiempos de John Dewey a inicios del siglo XX, se propone organizar un currículo experiencial a partir de proyectos. Además, hay una segunda característica de los currículos de bachillerato no abordada por la crítica: estos siguen adheridos al ideal positivista de la Escuela Nacional Preparatoria del siglo XIX -que proviene de la Escuela Nacional Politécnica francesa de 1798 (Weiss y Bernal, 2013) - de que hay que enseñar primero las materias básicas como matemáticas, ciencias, filosofía y lengua, es decir, las más abstractas, antes de que los alumnos puedan abordar y comprender materias y temas más concretos y relacionados con la vida.

La resistencia a los cambios - como vimos en la Reforma Integral de la Educación Básica (Quiroz y Weiss, 2005)- proviene de la fuerza que tienen las disciplinas en la academia y en la formación del personal docente, pero sobre todo en los términos de contratación de los docentes como docentes especialistas (cf. también Tyack y Tobin, 1998; Quiroz, 1992; Terigi, 2012). Es por ello que los cambios curriculares profundos solo suelen ser posibles en modalidades nuevas y en los márgenes de los sistemas establecidos. Por ello el Telebachillerato Comunitario resulta relevante.

Como podemos apreciar en lo expuesto hasta aquí, entre el "currículum pensado y el currículum vivido" (como lo llama Furlán, 1998) o el "currículum propuesto y el real" (como lo llaman De Ibarrola, 2012 y Terigi, 2012) median las "estructuras institucionales del currículum": "la selección, contratación y 
condiciones de trabajo de los profesores"; "la asignación de tiempo de enseñanza"; "la asignación de espacios"; "la asignación de recursos didácticos"; "la formas de evaluación y de certificación de los aprendizajes logrados" (De Ibarrola, 2012). Estas estructuras -con excepción de la última, que no indagamos- son de gran importancia para enmarcar nuestros hallazgos.

\section{Hallazgos generales. Un nuevo tipo de servicio aceptado por los usuarios}

El Telebachillerato Comunitario (TBC) no es "Tele-"; se llama Telebachillerato por el antecedente de este tipo de servicio en el Telebachillerato veracruzano donde, inicialmente, se transmitían los módulos por la televisión estatal y porque la gran mayoría de las escuelas están ubicadas en Telesecundarias. Estas últimas, en rigor, tampoco son Telesecundarias, ya que funcionan la mayor parte del día sin televisor o videos; se basan más bien en exposiciones del maestro y el estudio de los alumnos en cuadernos de trabajo con ejercicios; esporádicamente usan algún video. La innovación importante del Telebachillerato Comunitario, en términos de recursos didácticos, son libros de texto gratuitos que no existen en otros bachilleratos. Lo "Comunitario" actualmente solo constituye una aspiración de contribuir al arraigo de los jóvenes en la comunidad y al desarrollo comunitario; asimismo, en algunos estados las aulas aún se ubican en instalaciones proporcionadas por la comunidad.

En su gran mayoría, los centros de Telebachillerato Comunitario se ubican en localidades rurales o rural-urbanas que son pequeñas, fuera de las cabeceras municipales y a más de cinco kilómetros de distancia de otros servicios. Aunque también encontramos TBCs en espacios urbanos periféricos en el estado de México y en Aguascalientes. En este sentido, el nuevo tipo de servicio aparece no solo como una oferta que acerca la educación a aquellos lugares que no tienen otra opción, sino como la oferta que atiende a los que ya no pueden ser absorbidos por otros espacios. Por otro lado, en algunos estados (p. ej. Estado de México y Chihuahua) se observa una baja en la inscripción de los alumnos en primer semestre y un número apreciable de centros con menos 
de 12 alumnos en primer semestre, lo que parece indicar ciertas dificultades para extender más el servicio.

El estudio exploratorio muestra que el Telebachillerato Comunitario (TBC) es un servicio que contribuye a fortalecer el derecho a la educación, pues permite a los jóvenes asistir a la Educación Media Superior. Ellos acuden a los Telebachilleratos Comunitarios por las siguientes razones:

a) por su cercanía y la mayor seguridad en los caminos;

b) por su gratuidad. El costo del pasaje a otros planteles es de entre 16 y 32 pesos diarios (en 2015); en otros planteles el costo de libros y fotocopias llega a $\$ 400.00$; y en otros la cuota de cooperación para inscribirse es de aproximadamente $\$ 300.00$.

c) por sentirse tomados en cuenta en una escuela con pocos alumnos y maestros:

Ya le entiendo más al inglés; antes, en la secundaria, no le entendía nada, y si no le entendemos bien, nos lo vuelven a explicar. (Estudiante del TBC Mayorazgo, Estado de México).

Aquí me siento más a gusto, más cómoda, que allá, en el CETIS, éramos más de 50 alumnos y no nos ponían la suficiente atención y aquí (en el TBC) siento que las clases son más fáciles. (Estudiante del TBC La Quemada, Chihuahua).

Casi todos los alumnos provienen de la telesecundaria del lugar o de lugares cercanos. En la primera generación se hizo promoción con jóvenes de la comunidad que habían dejado sus estudios, pero dado que muchos de ellos abandonaron pronto, en las siguientes generaciones se promovió entre los egresados de telesecundaria. Pocos alumnos realizan un trabajo remunerado además de estudiar; una parte considerable ayuda en las actividades económicas y domésticas de sus familias, muy pocos de ellos en actividades agropecuarias. La mayoría de los estudiantes tiene deseos de seguir estudiando, algunos con mucha seguridad, otros "tal vez y si se puede".

Una parte de los docentes son jóvenes profesionistas con poco tiempo de haber egresado de licenciaturas o ingenierías. Otros ya 
tienen algunos años de trabajar como docentes en la educación media superior o secundaria en las mañanas. La mayoría vive cerca de la localidad en la que labora. La irregularidad del pago ha causado movilidad de aquellos docentes que tienen el TBC como única fuente de trabajo, situación que parece ser temporal, dada la regularización de los pagos mediante el Servicio Profesional Docente.

\section{Un nuevo tipo de servicio interesante en términos educativos}

El Telebachillerato es un nuevo tipo de servicio educativo con características interesantes.

Proporciona libros de texto gratuitos, lo que significa un gran ahorro para las familias de los estudiantes, al no tener que gastar en la compra de libros de texto o en las fotocopias que se usan en otros bachilleratos. A los docentes -en su mayoría jóvenes, provenientes de diversas profesiones (ingenieros, contadores, abogados, psicólogos, etc.- ofrece material educativo valioso para orientar sus clases, ya que ellos no saben qué contenidos, a qué nivel y con qué extensión son adecuados para las diferentes asignaturas que tienen enseñar, ni saben qué actividades a desarrollar con los alumnos son pertinentes -más allá de las que han vivido o sufrido en su propia escolarización-. Los libros de texto incluso son usados (en su versión electrónica) por maestros que ya tienen experiencia en otros bachilleratos para sus clases en esas escuelas. Para los alumnos, estos libros favorecen el trabajo con materiales escritos pertinentes y no solo a partir de exposiciones orales de los docentes e información de dudosa calidad encontrada en otros lados.

En su acervo, el Telebachillerato cuenta con 199 videos, sin embargo, su uso real es escaso, ya que hay problemas de infraestructura tecnológica (electricidad, conectividad, acceso a reproductor de DVD y pantallas) y el peligro de robo de los equipos. Un hallazgo sorpresivo y a tomar en cuenta es que, en comparación con los videos de la televisión y de los que circulan en las redes sociales, los estudiantes consideran anticuadas la imágenes y ritmos de los DVDs del TBC. Por ello algunos docentes bajan videos más idóneos de Internet. Curiosamente, esta objeción no 
se escucha con relación con los libros de texto, que son un objeto cultural poco conocido en las comunidades.

Otra innovación curricular interesante es el perfil de los docentes como docentes por campo disciplinar o área de conocimiento. En localidades pequeñas y lejanas, se dificulta tener profesores para todas las diversas asignaturas en tiempo cortos, como en las escuelas de bachillerato usuales. Por ello se recurrió a un modelo que ha sido propuesto reiteradas veces por pedagogos en todo el mundo. Entre 1978 y 1993 ya existía en México como opción para las escuelas secundarias y para la formación de profesores en las normales superiores: el área de conocimiento (cf. Quiroz y Weiss, 2005). El modelo del TBC instituyó tres campos disciplinares, cada uno a ser atendido por un docente: a) matemáticas y ciencias experimentales (matemáticas, estadística, física, química, biología, ciencias de la salud); b) comunicación (lectura y redacción, literatura, inglés); y c) ciencias sociales y humanidades (ética y valores, derecho y filosofía, historia de México y universal, estructuras socioeconómicas, metodología de investigación, desarrollo comunitario).

El diseño por campos disciplinares permite (potencialmente) superar la fragmentación excesiva del tiempo de docencia y de la experiencia de los alumnos del modelo de enseńanza por asignaturas con sus clases de 50 minutos (Quiroz, 1992). Al contar en el TBC, generalmente con grupos pequeños de 10 a 20 atendidos por los tres maestros, los docentes tienen mayores posibilidades de conocer bien a cada estudiante y de dar explicaciones más amplias.

\section{Las tensiones principales en el modelo y las estructuras institucionales del currículum}

Las principales tensiones que detectamos en el modelo y en su operación son: a) la ubicación de las escuelas en instalaciones de telesecundaria y en el contraturno vespertino, b) la sobrecarga de trabajo para tres maestros, de los cuales uno es responsable del servicio; c) las tensiones en el diseño curricular.

Compartir las instalaciones de las telesecundarias en el contraturno (en la tarde) significa un ahorro importante en infraestructura. Sin embargo, propicia en varias escuelas conflictos con 
el personal de la Telesecundaria y restricciones en el uso de las instalaciones, servicios, mobiliario y recursos tecnológicos (aulas, sanitarios, televisores, armarios, bancas, Internet, pantallas, DVDs); en muchas escuelas han buscado superar estos problemas, por ejemplo, en Guanajuato se establecieron convenios de buena convivencia.

Pero sobre todo, el contraturno, que obliga a los Telebachilleratos a funcionar en horario vespertino, oficialmente de 14:20 a 20:10 horas. Como en los semestres invernales oscurece alrededor de las 18:00 horas, en algunos planteles la iluminación es insuficiente y hay problemas o temores de inseguridad en los caminos, gran parte de los centros funcionan realmente de 14:30 a 18:00 horas, como reconocen las autoridades del Estado de México y de Chihuahua (estado en el que oscurece en invierno a las 17:00 horas), lo que constituye una disminución importante del tiempo escolar, comparado con planteles de bachillerato que funcionan en horario matutino de 8:20 a 14:10 horas. Por esta razón, las autoridades en Chihuahua y Veracruz han promovido que los Telebachilleratos cuenten con instalaciones propias y funcionen en horario matutino, aunque hay que señalar que algunas de estas instalaciones son aún precarias.

En discursos oficiales se justifica el turno de la tarde para dar cabida a estudiantes que trabajan y que, de otra manera, no podrían asistir a la educación media superior de carácter obligatorio. Sin embargo en los planteles visitados hemos encontrado pocos estudiantes que trabajan.

El modelo prevé para cada plantel tres maestros con 20 horas de contratación, uno de los cuales funge, a la vez, con 10 horas adicionales como responsable del plantel: lo llaman el modelo 20-20-30. El modelo funciona muy bien cuando los planteles son de reciente creación y solo tienen que atender un grupo de primer semestre o un grupo de primer semestre y otro de tercero. En los inicios de los establecimientos, los maestros tienen tiempo para familiarizarse con el modelo, los programas y los libros, realizar tutorías con los estudiantes o actividades paraescolares. Sin embargo, cuando los centros funcionan ya con tres semestres por atender a la vez, se genera una sobrecarga a todos los docentes con respecto al número de horas contratadas. Por ejemplo, 
cuando se tiene que atender en paralelo a $1^{\circ}, 3^{\circ}$ y $5^{\circ}$ semestres, el docente encargado de matemáticas tiene que impartir, según el plan de estudios, 33 horas de clase (de 50 minutos), es decir 27 horas y 30 minutos de enseńanza, a pesar de ser contratado solo por 20 horas.

Con la escuela funcionando con todos los semestres previstos, la sobrecarga se presenta también para los otros dos docentes, quienes tienen que impartir, según el plan, 28 horas clase $\left(24^{\circ}, 10^{\prime}\right)$ y 27 horas clase $\left(22^{\circ}, 30^{\prime}\right)$ respectivamente. Razón por la que las autoridades del Estado de México plantean la necesidad de un cuarto docente y en Guanajuato lo pagan con recursos estatales. Ante esta condición, proponen separar el área de matemáticas (en muchos casos atendida por Contadores) del área de ciencias exactas. La cuarta persona cumpliría también una función importante en caso de ausencia de alguno de los docentes, p. ej., por enfermedad o diligencias que tiene que realizar el responsable del plantel.

En los hechos, los profesores no se ciñen estrictamente al perfil de las asignaturas a impartir por área disciplinar, pues entre ellos buscan equilibrar sus cargas docentes. También porque determinados maestros cuentan con mayor conocimiento para responsabilizarse de alguna asignatura en particular, por ejemplo, el inglés, o bien, contadores de profesión nombrados para el área de matemáticas y ciencias, a quienes se les dificulta enseñar las ciencias experimentales.

Queda sin definición clara cuántas son las horas al día que se deben cubrir en los TBC: seis, como se estipula en las Guías para la práctica docente del TBC y en el Documento Base del Telebachillerato Comunitario (SEMS, 2015, 2015b), o cinco, como escuchamos en varios estados y como encontramos en los ejemplos de horarios que presenta la DGB en el Documento Base del TBC en su versión de 2015, o cuatro horas como encontramos en planteles en dos estados.

\section{Tensiones en el diseño curricular}

El modelo del Telebachillerato presenta la innovación de una formación interdisciplinaria por área de conocimiento y contra- 
tó docentes por campos disciplinares, además de proponer que buena parte de la formación general se realice mediante proyectos formativos interdisciplinarios. Sin embargo, el plan y programa de estudios, así como los libros de texto siguen organizados por las asignaturas convencionales de un bachillerato general. De hecho, los maestros enseñan apoyados en los libros de texto por asignatura. Asimismo, el modelo enfatiza la formación para el trabajo mediante proyectos de desarrollo de la comunidad. Sin embargo, el número de horas destinadas a los proyectos es limitado y su orientación e implementación son deficientes como veremos más adelante.

El plan de estudios del Telebachillerato Comunitario es el plan de la Dirección General de Bachilleratos (DGB), que es usado también en otros servicios, incluyendo el Colegio de Bachilleres. Es el plan de un bachillerato general fuertemente propedéutico con un carácter enciclopédico que fue conformado en el transcurso del siglo XX y que se basa esencialmente en las disciplinas académicas.

El plan sigue siendo el mismo de 1995 (las mismas materias, los mismos contenidos), solo revisado y ajustado superficialmente en términos del enfoque de competencias que propuso la Reforma Integral de la Educación Media Superior. Así, mientras el modelo curricular proclama la interdisciplinariedad y el trabajo por proyectos, en realidad sigue siendo un plan de estudios de colección de asignaturas (como lo llama Bernstein) con su típica orientación positivista de origen politécnica (cf. Weiss y Bernal, 2013), primero las matemáticas y las ciencias, algo de filosofía, lenguas e historia y al último ciencias de la vida y desarrollo comunitario.

A pesar del poco énfasis que en la propuesta curricular se hace en las actividades paraescolares, encontramos que en algunas escuelas los maestros las promueven (actividades deportivas y culturales y talleres de informática) y son muy apreciadas por los alumnos. Los docentes buscan ofrecerlas de acuerdo con sus habilidades y conocimientos y según el tiempo disponible. 


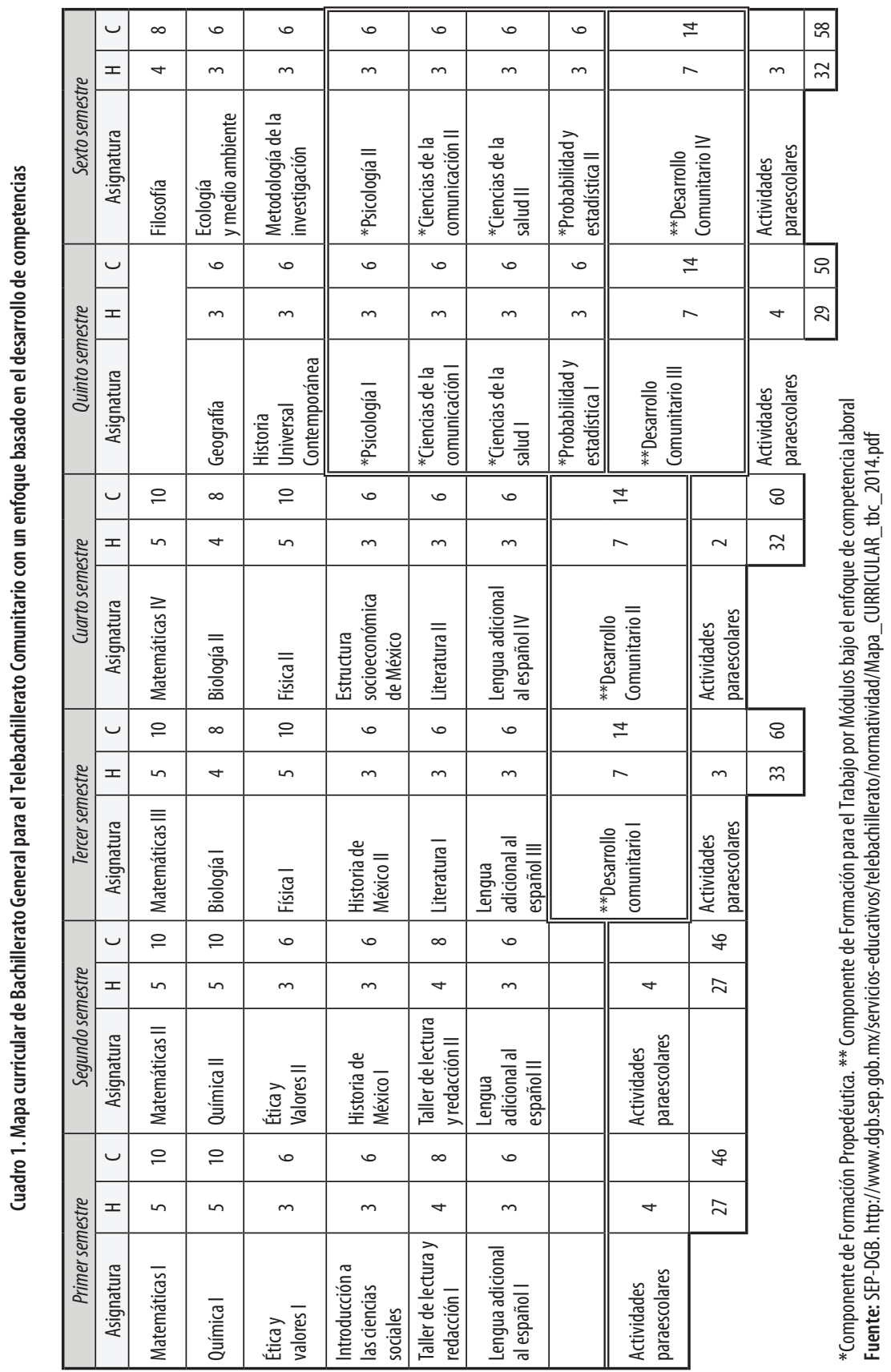


El pequeño componente de formación para el trabajo que aparece también en otros bachilleratos generales -más como legitimación que como intento serio- se dedica en el TBC al "Desarrollo comunitario". Se aborda solo del tercer al sexto semestre y se atiende en siete de las 25 a 30 horas de clase semanales. Tenemos la impresión que son las horas que más se recortan en la práctica.

El trabajo por proyectos de desarrollo comunitario es incipiente o inexistente en los planteles. Escuchamos discursos de algunas autoridades respecto a la contribución de los proyectos de desarrollo comunitario en términos de emprendimientos y proyectos productivos que puedan fomentar el desarrollo de las comunidades y proveer trabajo a los egresados del TBC. Por lo que conocemos de las dificultades para armar proyectos productivos y de desarrollo comunitario en ámbitos escolares -véase Weiss (1991) sobre la dificultades en bachilleratos tecnológicos agropecuarios y Weiss (2006) sobre los Bachilleratos Interculturales Comunitarios de Oaxaca que cuentan con brigadas de extensión- vemos que estos discursos son poco realistas, máxime en escuelas que no tienen docentes con experiencias profesionales productivas y comunitarias.

Además, el "Programa de Estudios para el Desarrollo Comunitario" que ofrece la Subsecretaría (SEMS, 2015b) es excesivamente académico. Prevé, para el tercer semestre, estudiar los significados del desarrollo comunitario y de la comunidad, para el cuarto elaborar un diagnóstico comunitario, apenas en el quinto diseñar y poner en práctica un proyecto y en el sexto evaluarlo y darle seguimiento. Es decir, el diseño y la puesta en práctica de un proyecto se limita al $5^{\circ}$ semestre y los restantes se dedican a conceptualizaciones, diagnósticos y evaluaciones. Asimismo el diseńo se mete en complicaciones al tratar de fomentar en paralelo proyectos interdisciplinarios y comunitarios.

De hecho, en las escuelas visitadas, casi todos los proyectos, aun en quinto semestre, se encontraban todavía en estado de planeación. Algunas propuestas resultan interesantes, unas inclinándose más al servicio comunitario, como una guardería o un comedor. 


\section{Conclusiones}

El Telebachillerato Comunitario ofrece, por su cercanía y gratuidad en comunidades rurales y rural-urbanas, acceso a la educación a estudiantes que, de no existir esta opción (o una similar como la Educación Media Superior a Distancia EMSAD o el Bachillerato Intercultural), probablemente ya no asistirían a las escuelas. Tiene aceptación por las familias que aprecian la cercanía y la gratuidad efectiva y por los estudiantes que se sienten atendidos en grupo pequeños por un número reducido de maestros que los conocen y los atienden.

Las innovaciones curriculares más importantes son a) los libros de texto gratuitos que bajan los costos de la escolaridad, orientan a los docentes jóvenes provenientes de diversas profesiones en qué y cómo enseñar y permiten a los alumnos trabajar con material escrito de buena calidad; b) la contratación de docentes por campo disciplinar, lo que reduce el número de docentes y aumenta el tiempo de trabajo conjunto de maestros y alumnos.

La deficiencia más importante es el número reducido de horas clase, en muchas escuelas trabajan de las 14:00 a las 18:00 horas de la tarde. Esta reducción a cuatro horas, que disminuye las experiencias de aprendizaje en comparación con las seis horas usuales en los bachilleratos, tiene dos orígenes: a) no contar con instalaciones propias, el Telebachillerato se imparte en las horas contraturno vespertinas y en muchas comunidades consideran inseguros los caminos después de las 18:00 horas; b) los docentes solo tienen 20 horas de contrato, que se cumplen en 5 días $\mathrm{x} 4$ horas. Para superar estas deficiencias se requieren instalaciones propias (como las están construyendo en algunos estados) y la contratación de un cuarto docente (como lo hacen en otros). Ambas soluciones requieren más financiamiento, pero son necesarias si se quiere atender, cuando menos con la misma calidad, a la población rural vulnerable.

De otros estudios sabemos que en el camino de innovaciones educativas desde las políticas educativas y modelos curriculares hasta las realidades escolares ocurren necesariamente sucesivas "recontextualizaciones" (Ezpeleta, 2004). Sin embargo, la contradicción curricular entre la contratación de docentes por campo disciplinar y el plan de estudios tradicional por asignaturas tendrá 
que superarse por un plan de estudios más integral diseñado por áreas. Curiosamente, este tipo de plan se instauró para otra innovación curricular en el mismo sexenio, la "Prepa en línea" que inició en 2014 (Diario Oficial de la Federación 29 de septiembre de 2014), más no se usó para el Telebachillerato Comunitario que se instauró un año antes. A la vez, habrá que fortalecer los proyectos comunitarios y temas relevantes para los jóvenes y superar la orientación academicista que concibe los proyectos como problemas de investigación, en lugar de como tareas a planear y realizar. Estos pueden comenzar desde el primer semestre en forma práctica, si se supera el paradigma positivista de que primero hay que enseñar conceptos abstractos, antes de poder abordar temas concretos.

\section{Referencias bibliográficas}

Banks, J. Educating Citizens in a Multicultural Society, Nueva York, Teachers College Press, 2007.

Bellei, C. "Políticas educativas para el nivel secundario: complejidades y convergencias", en E. Tenti Fanfani (Ed.) La escolarización de los adolescentes: desafíos culturales, pedagógicos de Investigación en Juventud y de politica educativa, Buenos Aires, IIEP-UNESCO, 2012, 209-253.

CONAEDU. XLII Reunión del Consejo Nacional de Autoridades Educativas, 2015. Disponible en http://www.gob. $\mathrm{mx} / \mathrm{sep} /$ documentos/xlii-reunion-conaedu-ems-parte1 ?state=published. Fecha de consulta 20 abril de 2017.

De Ibarrola, Ma. "Experiencias y reflexiones sobre el diseño y la evaluación curricular", en Monique Landesmann. El curriculum en la globalización. A tres décadas de "El curriculum pensado y el curriculum vivido", México, UNAM, 2012, 93-118.

DGB. "El Telebachillerato Comunitario", presentación realizada en el Instituto Nacional para la Evaluación de la Educación, septiembre de 2015. Dirección General de Bachillerato. Disponible en http://www.dgb.sep.gob.mx/ servicios-educativos/telebachillerato/index.php. Fecha de consulta 20 abril de 2017. 
Diario Oficial de la Federación, México, 9 de febrero de 2012. Disponible en http://www.iea.gob.mx/webiea/emys/archivos/decreto_educacion_media.pdf. Fecha de consulta 20 de abril de 2017.

Diario Oficial de la Federación, México, 29 de septiembre de 2014. Acuerdo número 09/09/14 por el que se establece el Plan de Estudios del Servicio Nacional de Bachillerato en Línea, Prepa en Línea-SEP. Disponible en http:// dof.gob.mx/nota_detalle.php?codigo $=5361362 \& \mathrm{fec}$ ha $=24 / 09 / 2014$. Fecha de consulta 20 de abril de 2017 .

Ezpeleta, J. "Innovaciones educativas. Reflexiones sobre los contextos en su implementación", en Revista Mexicana de Investigación Educativa, vol. 9, núm. 21, 2004, pp. 404-423.

Furlán, A. "Currículum y condiciones institucionales", en Currículum e institución, Morelia, México, Instituto Michoacano de Ciencias de la Educación, 1998, 89-102.

INEE. La educación media superior en México. Informe 20102011, México, Instituto Nacional para la Evaluación de la Educación, 2011. Disponible en http://publicaciones. inee.edu.mx/buscadorPub/P1/D/235/P1D235.pdf.Fecha de consulta 20 de abril de 2017.

Pérez Islas et al. "Evaluación de la política contra la interrupción escolar en la educación media superior", Informe para el INEE, Seminario UNAM, México, 2016.

Quiroz, R. "El tiempo cotidiano en la escuela secundaria”, en Nueva Antropología, núm. 42, 1992, 89-100.

Quiroz, R. y Weiss, E. "Balance y Perspectivas de la Reforma de la Educación Secundaria en México", en E. Weiss, R. Quiroz y A. Santos del Real, Expansión de la educación secundaria en México. Logros y dificultades en eficiencia, calidad y equidad, París, IIPE-UNESCO, Serie Políticas y estrategias para la educación secundaria, 2005, 93-156.

Sadovnik, A. "Basil Bernstein (1924-2000)", Perspectivas: revista trimestral de educación comparada, París, UNESCO, XXXI, 4, 2001, 687-703.

SEMS. Documento Base. Telebachillerato Comunitario, México, Subsecretaría de Educación Media Superior, 2015. Disponible en http://www.dgb.sep.gob.mx/servicios-educativos/telebachillerato/normatividad/DOCUMENTO_ BASE_TBC_2015.pdf. . Fecha de consulta 20 abril de 2017. 
SEMS. Desarrollo Comunitario, Telebachillerato Comunitario. Tercer y Cuarto Semestres, México, Serie Programas de Estudio, Secretaría de Educación Pública/Subsecretaría de Educación Media Superior, 2015b. Disponible en http:/www.dgb.sep.gob.mx/servicios-educativos/telebachillerato/LIBROS/3-semestre-2016/Desarrollo-Comunitario-III-y-IV.pdf. Fecha de consulta, 20 de abril de 2017.

SEP. Cuarto Informe de Labores 2015-2016, México, Secretaría de Educación Pública, 2016. Disponible en http://www.planeacion.sep.gob.mx/Doc/informes/ labores/2012_2018/4to_informe_de_labores.pdf. Fecha de consulta, 20 de abril de 2017.

SITEAL. "Escolarización en América Latina 2000-2013. Resumen estadístico comentado", en Resúmenes estadísticos comentados. Sistema de Información de Tendencias Educativas en América Latina, Buenos Aires, IIPE-UNESCO, Sede Regional Buenos Aires-OEI, 2015. Disponible en http://www.siteal. iipe.unesco.org/sites/default/files/rec_siteal_2_2015_04_28. pdf. Fecha de consulta, 20 de abril de 2017.

Tenti, E. "Docentes y alumnos: encuentros y desencuentros entre generaciones", en E. Tenti Fanfani (Ed.) La escolarización de los adolescentes: desafíos culturales, pedagógicos y de politica educativa, Buenos Aires, IIEP-UNESCO, 2012, 191-208.

Terigi, F. "Sobre la cuestión curricular en la educación secundaria", en E. Tenti Fanfani (Ed.) La escolarización de los adolescentes: desafíos culturales, pedagógicos y de politica educativa, Buenos Aires, IIEP-UNESCO, 2012, 55-78.

Tyack, D. y W. Tobin. En busca de la utopía, México, Fondo de Cultura Económica, 1998.

Viñao, A. Sistemas educativos, culturas escolares y reformas. Continuidades y cambios, Madrid, Morata, 2002.

Weiss, E. Diagnóstico de las Prácticas y Procesos Curriculares en los Bachilleratos Integrales Comunitarios, México, Coordinación General de Educación Intercultural Bilingüe, SEByN-SEP, Informe sin publicar, 2006.

Weiss, E. "El abandono escolar en la educación media superior: dimensiones, causas y políticas para abatirlo”, en Ramí- 
rez, Rodolfo (coord.) Desafíos de la educación media superior, México, Instituto Belisario Domínguez del Senado de la República, 2015, 81-159.

Weiss, E. "La formación escolar del técnico agropecuario en México 1970-1990", en Comercio Exterior, México, Banco Nacional de Comercio Exterior, 41, núm. 1, 1991. 68-78. Weiss, E. y E. Bernal "Un diálogo con la historia de la educación técnica mexicana”, en Perfiles Educativos, México: CESUUNAM, XXXV, núm.139, 2013, 151-170.

Zorrilla, J. "Prioridades formativas para el bachillerato mexicano. La agenda mínima”, en Perfiles Educativos, México, CESU UNAM, XXXIV, número especial, 2012, 70-83. 